\title{
The Neurophysiology of Figure-Ground Segregation in Primary Visual Cortex
}

\author{
Victor A. F. Lamme \\ The Department of Brain and Cognitive Sciences, Massachusetts Institute of Technology, Cambridge, Massachusetts \\ 02139
}

\begin{abstract}
The activity of neurons in the primary visual cortex of the awake macaque monkey was recorded while the animals were viewing full screen arrays of either oriented line segments or moving random dots. A square patch of the screen was made to perceptually pop out as a circumscribed figure by virtue of differences between the orientation or the direction of motion of the texture elements within that patch and the surround. The animals were trained to identify the figure patches by making saccadic eye movements towards their positions. Almost every cell gave a significantly larger response to elements belonging to the figure than to similar elements belonging to the background. The figure-ground response enhancement was present along the entire extent of the patch and was absent as soon as the receptive field was outside the patch. The strength of the effect had no relation with classical receptive field properties like orientation or direction selectivity or receptive field size. The response enhancement had a latency of $\mathbf{3 0 - 4 0 ~} \mathrm{msec}$ relative to the onset of the neuronal response itself. The results show that context modulation within primary visual cortex has a highly sophisticated nature, putting the image features the cells are responding to into their fully evaluated perceptual context.
\end{abstract}

[Key words: figure-ground segregation, primary visual cortex, awake macaque monkey, single-unit activity, texture, motion, context modulation, visual perception]

The segregation of a visual scene into figure and ground belongs to the most fundamental of problems that have to be solved by the visual system. Its neurophysiological correlates are, however, still obscure. The early stages of visual processing, in the retina, LGN, and primary visual cortex, are characterized by small receptive fields. Here, elementary features such as local luminance or chrominance contrast seem to be extracted from the image (Lennie, 1980; Livingstone and Hubel, 1988) followed by extraction of the orientation, direction of motion (Hubel and Wiesel, 1959, 1977; Schiller, 1976), and disparity (Poggio and Fischer, 1977) of contrast. To segment an image into figure and

\footnotetext{
Received Dec. 15, 1993; revised July 21, 1994; accepted Aug. 5, 1994.

This research was supported by a grant from The Netherlands Organization for Scientific Research (NWO) to V.A.F.L. and by an NEI (NIH) grant to Peter H. Schiller, in whose laboratory this work was carried out. I thank Peter H. Schiller, Karl Zipser, and Warren M. Slocum for their helpful suggestions in preparing the experiments.

Correspondence should be addressed to Victor A. F. Lamme, Graduate School Neurosciences Amsterdam, The Netherlands Ophthalmic Research Institute, P.O. Box 12141, 1100 AC Amsterdam, The Netherlands.

Copyright (c) 1995 Society for Neuroscience 0270-6474/95/151605-11\$05.00/0
}

background the features belonging to a circumscribed object have to be grouped and separated from groups of features belonging to other objects or to the background. Classically, only a subordinate role is allocated to primary visual neurons in this process. They are thought to provide only the input to higher order areas, where the extraction of meaningful feature combinations supposedly occurs (Barlow, 1972; Perret et al., 1987). Neurons in the higher cortical areas do have larger receptive fields, but, with the longstanding exception of cells responding preferentially to aspects of faces and hands (Gross et al., 1972; Rolls, 1991), the features that trigger these neurons remain clusively abstract (Maunsell and Newsome, 1987).

Alternatively, feature grouping might not be reflected in the receptive field characteristics of any single neuron but might instead be mediated by the cooperative activity of ensembles of feature selective neurons throughout the visual brain. Synchronization of neuronal firing has been proposed as a mechanism for feature linking (Milner, 1974; Von der Malsberg and Schneider, 1986) and recent evidence has provided support for this idea (Eckhorn et al., 1988; Gray et al., 1989).

In addition, it has often been observed that the neurons in the early stages of processing (and beyond) can be influenced by image attributes that are far away from the "classical" receptive field itself. When the receptive field is stimulated with an appropriate stimulus the presentation of stimuli in the receptive field surround may modulate its response. A typical property is that these surround stimuli never evoke a response when presented alone. A variety of effects have been reported ranging from quite unspecific inhibition or facilitation to orientation or direction selective effects (e.g., Allman et al., 1985a, for a review, and see Discussion).

The function of these context modulation effects is still not very clear. Some pertinence to global image processes such as color or texture constancy, the motion aperture problem, texture segregation or figure-ground segregation has been suggested. This could however not be ascertained because of the receptive field centered nature of the experiments. Typically, the receptive field was stimulated with an optimal stimulus and the effect of various surround stimuli was determined. The results could therefore not be interpreted beyond showing the existence of lateral interactions per se.

In this study the receptive fields of neurons in the striate cortex of the macaque monkey were stimulated with textured arrays consisting of oriented line segments or moving random dots. They were made to appear either as background or as figures by virtue of differences in the orientation or the motion of image features in surrounding portions of the visual scene. Almost every cell gave a significantly larger response for texture ele- 
ments that were perceived as a figure than for background elements. To study the precise role of these effects in the segregation of figure and ground, the responses of the cells were analyzed with the receptive field at various positions relative to the border of figure and ground, leaving stimulation of the receptive field identical in all cases. It was observed that the response enhancement was only present for positions where the receptive field was within the houndaries of the figure and was absent for positions outside of the figure, even when close to the boundary between figure and ground. This asymmetry of the effect across the boundary between figure and ground shows that the lateral interactions observed are not simply due to lateral inhibition or other mechanisms related to the detection of edges. Instead, it appears that context modulation within a cortical area as early as V1 puts the features the cells are responding to into their perceptual context of figure and ground.

\section{Materials and Methods}

Display and recording equipment. Stimuli were presented on an NEC multisync XL color video display unit, driven by a Number Nine Corporation graphics board with a $640 \times 480$ pixel resolution, at a frame rate of $60 \mathrm{~Hz}$. The screen was $32 \times 24 \mathrm{~cm}$ and was viewed from a distance of $58 \mathrm{~cm}$. One pixel thus corresponded to a visual angle of $0.05^{\circ}$, and full screen size was $32^{\circ} \times 24^{\circ}$.

Recordings were made transdurally with glass coated platinum-iridium microelectrodes through a surgically implanted well overlying the operculum of area 17 . Impedance of the electrodes ranged from 0.5 to 3.0 M 2 . Spikes from single units or in some cases clusters of several units were isolated by setting an amplitude threshold.

All surgical procedures were performed under deep pentobarbital anesthesia and all experimental procedures were in accordance with NIH guidelines (see also Haenny and Schiller, 1988).

Visual stimulation and behavioral paradigm. Prior to recording the monkeys were habiluated to the laboratory environment and to seating in the primate chair with their heads restrained. Subsequently, the animals were trained to maintain fixation (within a window of $1.0^{\circ} \times 1.0^{\circ}$ ) at a small red spot $\left(0.2^{\circ}\right.$ diameter $)$ and to make saccadic eye movements to various targets as soon as the red fixation spot had disappeared. Eye movements were recorded using implanted scleral search coils (Robinson, 1963; Schiller et al., 1990) and digitized at a rate of $200 \mathrm{~Hz}$. Correct saccades were rewarded with drops of apple juice. When the monkey had acquired a sufficient level of performance the recording well was implanted.

To assess the receptive field characteristics of isolated units, black bars of light were moved over a homogeneous white background while the monkey maintained fixation for periods of $1-4 \mathrm{sec}$. In these experiments, the saccade target typically consisted of a red circular patch of $1.2^{\circ}$ diameter which randomly appeared at one out of four positions. Size, position, and speed of movement of the bar were optimized before a series of eight orientations were tested to determine the orientation and direction selectivity of the cell. In addition, the cell's preference was tested for the range of random dot and oriented line segment patterns that was used in this study. The complete set of available stimuli was limited to the combinations of two random dot sizes $\left(0.05^{\circ}\right.$ and $0.1^{\circ}$, density $50 \%$ ) with four directions of motion $\left(45^{\circ}, 135^{\circ}, 225^{\circ}\right.$, and $315^{\circ}$ ), and two types of randomly positioned line segments $\left(0.05^{\circ} \times\right.$ $0.2^{\circ}$, density $25 \%$ and $0.1^{\circ} \times 0.4^{\circ}$, density $16.7 \%$ ) with four orientations $\left(0^{\circ}, 45^{\circ}, 90^{\circ}\right.$, and $\left.135^{\circ}\right)$. A $4^{\circ} \times 4^{\circ}$ pattern patch overlying the center of the cell's receptive field was moved (in case of the random dot patterns) or flipped orientation (in case of the line segment patterns) to evoke a response. About $40 \%$ of cells encountered sufficiently responded to at least one of these stimuli. Typically, only the pattern the cell responded best to was used for the figure-ground experiments (see below), but occasionally more than one was used.

Two types of displays were used in the figure-ground experiments In the first, figure and ground were defined by differences in the direction of motion of random dots, in the second by differences in the orientation of randomly positioned line segments. Figure 1 depicts the paradigm of the motion display. A $4^{\circ} \times 4^{\circ}$ square window of the screen was positioned so that the receptive field of the cell was in its center. A similar window was positioned at the same eccentricity and elevation in the contralateral hemifield. At the beginning of each trial a small $\left(0.2^{\circ}\right.$ diameter) red fixation spot appeared in the center of the screen that was filled with random dots. After the monkey had fixated for $300 \mathrm{msec}$ the dots inside the square windows were moved independently of the dots covering the remainder of the screen. The dots started to move at the beginning of the "figure appears" period (time instance 0 ) and stopped moving after $67 \mathrm{msec}$ (i.e., four frames). Within this interval they were displaced over a distance of 2 pixels (one pixel at frame 0 and the second at frame 3 ). So during the short motion phase the speed of movement was 2 pixels per $67 \mathrm{msec}$ or 30 pixels per second (i.e., $1.5^{\circ}$ or $3.0^{\circ}$ of visual angle per second, depending on the random dot pixel size used). Four stimuli were used (Fig. $1 A-D$ ), combining two directions of motion, one of which optimally stimulated the cell, the other $180^{\circ}$ opposite in direction (see above for limitations of the available ranges of directions of motion and sizes of the dots). For stimuli $A$ and $C$ motion within the window overlying the receptive field was in opposite direction to the motion of the background, whereas motion within the window in the contralateral hemifield was in the same direction as motion of the background. Therefore, in these cases the square overlying the receptive field perceptually popped out as a distinct figure while the other one merged with the background. In stimuli $B$ and $D$ the situation was reversed: motion within the window overlying the receptive field merged with the background and the window in the contralateral hemifield emerged as a figure by virtue of the opposite dircctions of motion of this window and the background. Note that stimulation of the receptive field was identical in cases $A$ and $B$ and in cases $C$ and $D$. The difference between $\mathrm{A}$ (or $\mathrm{C}$ ) and $\mathrm{B}$ (or D) was that the same moving features in the one case (A or $C$ ) represented a circumscribed figure but in the other case ( $B$ or $D$ ) represented background. The dots never surpassed the window boundaries, that is, motion discontinuity was always present at all borders.

A similar paradigm was employed for the oriented line segments displays (Fig. 2). Both windows and background were filled with randomly positioned line segments of a particular orientation. The line segments were clipped at the window borders. After the monkey had fixated a red spot for $300 \mathrm{msec}$ these sets of line segments were replaced by other sets, either of the same orientation (but with different random spatial order) or of an orientation at right angles to the initial one. Again, four stimuli were created, numbered $A$ to $D$ in Figure 2, using two orientations, one of which optimally stimulated the cell and the other at right angles. In stimuli $A$ and $C$ the window overlying the receptive field emerged as a figure by virtue of differences between the orientations of the line segments of window and background. In stimuli B and D the window in the contralateral hemifield was made to appear as figure and the window overlying the receptive field was made to merge with the background. Again, stimulation of the receptive field was identical in cases $A$ and $B$ and in cases $C$ and $D$. The difference between $A$ (or $C$ ) and $B$ (or D) was that the same oriented line segments in the one case ( $A$ or $C$ ) represented a circumscribed figure but in the other case (B or $\mathrm{D})$ represented background.

In addition to having the window centered over the receptive field, the stimuli were used with the windows at various (vertical) positions relative to the receptive field (Fig. 3). A range of positions with the receptive field within as well as outside of the window were used, provided the cell could be isolated for a long enough period.

The monkeys had to fixate the small red spot for $300 \mathrm{msec}$ after stimulus onset. Then, the fix-spot disappeared and the animals were allowed to make a saccadic eye movement to the position of the motion or orientation defined figure. In case of the motion stimulus, the dots moved to their original position at this instance. Trials with the "figure" on either the left or right side of the fix-spot were randomized. Correct responses were rewarded with apple juice. Alternatively, a $1.2^{\circ}$ diameter red spot appeared randomly at one out of four positions and the monkey had to make a saccadic eye movement to these targets to obtain a reward. The position of these targets was not correlated with the position of the motion or orientation defined figures. The two behavioral paradigms were interleaved in blocks of several tens of trials and the monkeys easily switched from one paradigm to the other within two or three trials.

Control experiments consisted of running the trials with only the background dots or line segments, so leaving the windows blank, or with only the window dots or line segments, so leaving the background blank. Obviously, in these cases the behavioral task was to make a saccade to the red targets.

Data analysis. The shown peristimulus time histograms were calcu- 

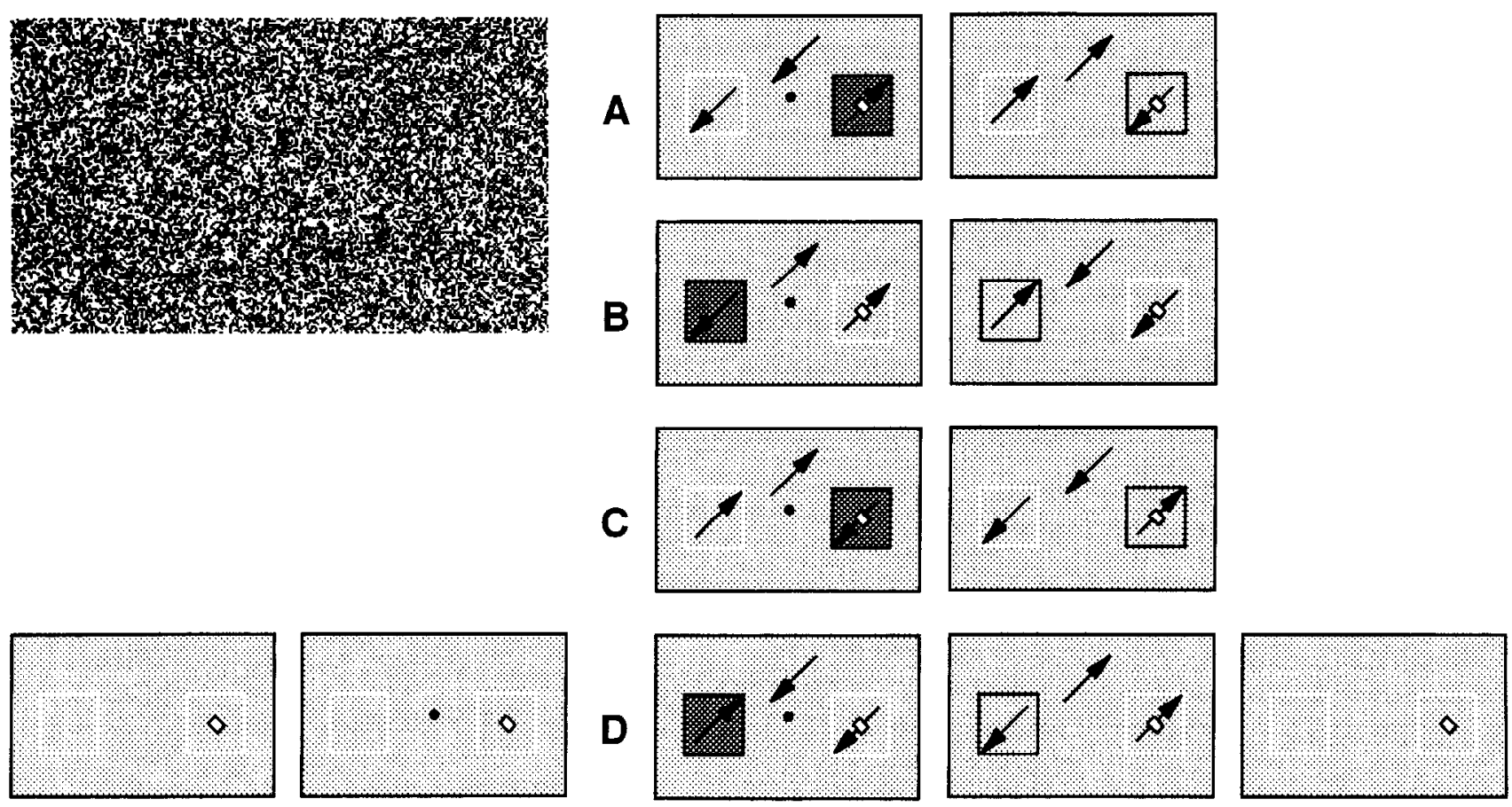

\begin{tabular}{|c|c|c|c|c|c|c|c|c|c|c|c|}
\hline \multicolumn{2}{|c|}{...Texture Screen } & \multirow[t]{2}{*}{1} & \multicolumn{2}{|c|}{ Fix-spot On } & \multirow[t]{2}{*}{1} & \multirow{2}{*}{ Figure Appears } & \multirow[t]{2}{*}{1} & \multirow[t]{2}{*}{ Fix-spot Off } & \multirow[t]{2}{*}{1} & \multicolumn{2}{|c|}{ Figure Disappears... } \\
\hline & + & & & & & & & & & + & 1 \\
\hline-600 & -450 & & -300 & -150 & 0 & 150 & 300 & 450 & 600 & 750 & 900 \\
\hline & & & & & & & & Saccade & 1 & Rewarded & \\
\hline
\end{tabular}

Figure 1. Prescntation scquence for onc completc trial of random dot motion figure-ground displays. Arrows indicate the directions of motion within the given windows. The small dot indicates the position of the fixation spot. The small open rectangle demarcates the position and approximate size of a putative receptive field under study. For illustration purposes the motion defined figure windows are shown darker than the background windows and white lines demarcate the windows. Above left, part of an actual display is shown. Below, a time axis is shown together with the timing of the stimulus events (top row) and the behavioral events (bottom row). The heavy part of the time axis demarcates the window of analysis for the PST histograms shown in the following figures. See Materials and Methods for further explanation.

lated off line, using bin widths of $30 \mathrm{msec}$ or $10 \mathrm{msec}$. Stimulus presentation and spike collecting synchronization was within $1 \mathrm{msec}$. Response strength is expressed in spikes per second. Spikes were sorted according to the different types of stimuli and correct completion of the trial. During the experiments, trials were blocked so that an equal number of correct trials per stimulus condition resulted. Standard deviations of histogram bins were calculated from the trial to trial variation.

Receptive field size was measured as the extent over which an optimally sized bar at the optimal position, orientation, speed, and direction elicited a response that was exceeding background levels. For this purpose a bell-shaped curve was hand-fitted to the peristimulus time histograms and the width of that curve was determined at $10 \%$ of the distance between its base and top. Orientation selectivity was measured as the total response elicited by the least effective orientation divided by the response elicited by the most effective orientation. Direction selectivity was measured as the response elicited by the nonpreferred direction at the optimal orientation divided by the response to the preferred direction. Lowest values thus indicate the strongest orientation and direction selectivity.

A figure versus background "modulation index" was calculated by dividing the sum of the total number of spikes between 0 and $300 \mathrm{msec}$ after stimulus onset of responses $A$ and $C$ by the sum of responses $B$ and $D[(\Lambda+C) /(B+D)]$. Standard deviations of modulation indices were calculated from the block to block variation where each block consisted of four sets of the four stimulus trials arranged in random order (this 4 $\times 4$ blocking was done to avoid predictability of one in every four trials, which might have been picked up by the monkeys).

To obtain results pooled from more than one neuron the numbers of spikes were averaged.

\section{Results}

Recording sites

About 200 single units or clusters of several units within primary visual cortex of two monkeys (Macaca mulatta) were isolated. Only those cells which responded sufficiently to at least one of the random dot or oriented line segment patterns were analyzed further. A total of 85 cells thus remained. The receptive fields were at eccentricities ranging from $3.3^{\circ}$ to $6.0^{\circ}$, in the right lower quadrant of the visual field. The animals have not yet been sacrificed but from the position of the well, in combination with the topography of the receptive fields, it could be concluded that the cells recorded from were in striate cortex.

\section{Figure-ground riodulation}

Figure 4 shows three examples of responses to the displays where figure and ground were defined by differences in the directions of motion (see Fig. 1). These results were obtained with the receptive field positioned in the center of the square window forming the figure. As explained in Materials and Methods, stimulation of the receptive field was identical in displays $A$ and $B$ and in C and D. In A and C, however, the moving dots within the window overlying the receptive field were made to appear as a distinct figure. In $\mathrm{B}$ and $\mathrm{D}$ the window in the contralateral 


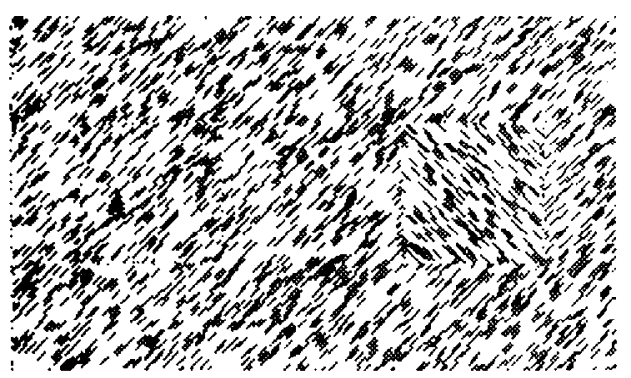

A
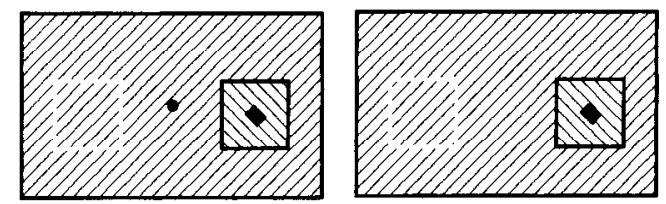

$\mathbf{B}$
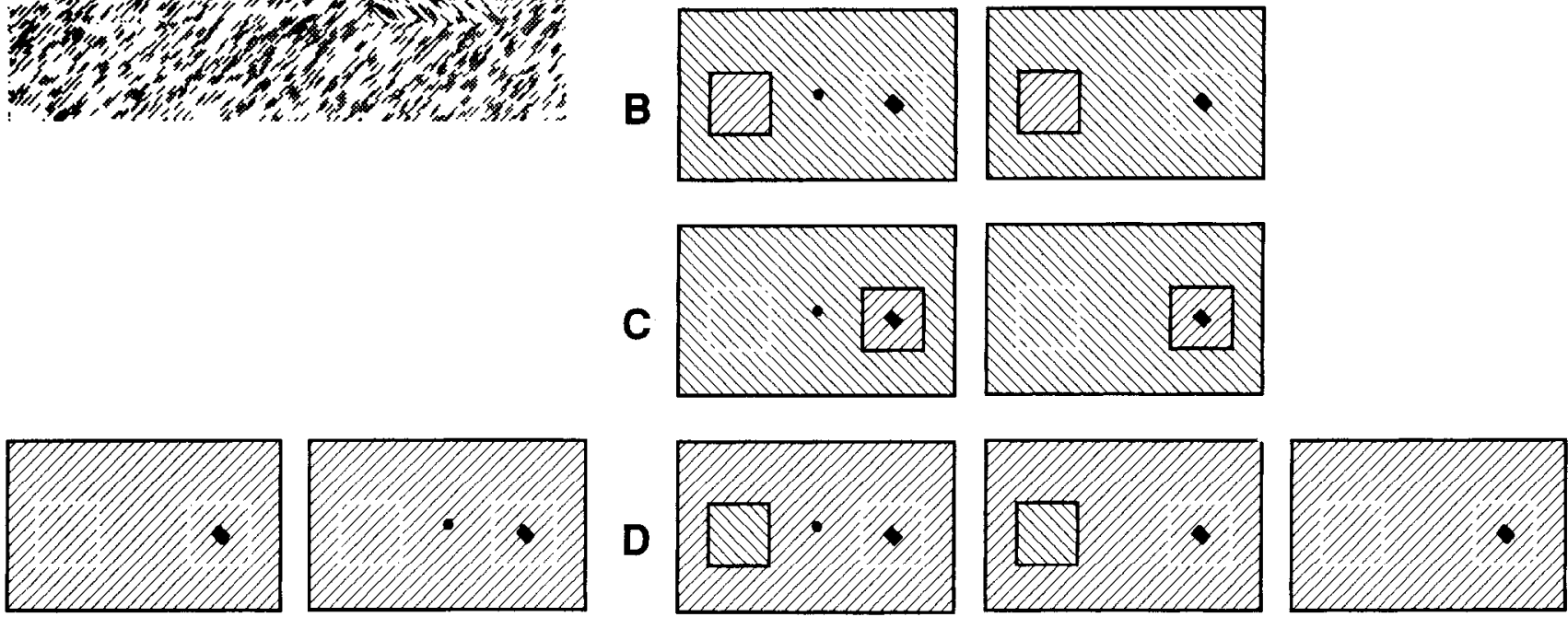

\begin{tabular}{|c|c|c|c|c|c|c|c|c|c|c|}
\hline ...Text & reen & Fix- & & 1 & Figure Appears & 1 & Fix-spot Off & 1 & \multicolumn{2}{|c|}{ Figure Disappears... } \\
\hline+ & + & & & & 1 & 1 & 1 & + & + & \\
\hline-600 & -450 & -300 & -150 & 0 & 150 & 300 & $\begin{array}{c}1 \\
450\end{array}$ & 600 & 750 & 900 \\
\hline & & & & & & & Saccade & 1 & Rewarded & \\
\hline
\end{tabular}

Figure 2. Presentation scquence for one complete trial of oriented line segment figure-ground displays. Orientation of the hatching indicates the orientation of the random line segments. Here, the receptive field is demarcated with a solid rectangle and the figure window is demarcated with a black outline. Above left, part of an actual display is shown. Below, a time axis is shown together with the timing of the stimulus events (top row) and the behavioral events (bottom row). The heavy part of the time axis demarcates the window of analysis for the PST histograms shown in the following figures. See Materials and Methods for further explanation.

hemifield was made to appear as a figure and the window overlying the receptive field merged with the background.

For all three cells shown the responses were larger when the moving dots that were overlying the receptive field constituted a figure as opposed to when the dots constituted background (compare $\mathrm{A}$ to $\mathrm{B}$ and $\mathrm{C}$ to $\mathrm{D}$ ). Cell 114 exhibited some direction selectivity; responses $A$ and $B$ were larger than responses $C$ and $D$, respectively. The figure-background enhancement effect was present for both the preferred and nonpreferred directions of motion.

Figure 5 shows three examples of responses to the displays where figure and ground were defined by differences in the orientation of line segments (see Fig. 2). These results were also obtained with the receptive field in the center of the window. Cell 129 responded only to stimuli $A$ and $B$, reflecting a strong orientation selectivity. The response was about twice as large when the line segments overlying the receptive field made up a figure (A) as opposed to when they made up the background (B). Cell 14 exhibited the strongest figure-background modulation observed. It responded strongly to stimulus $\mathrm{A}$, where the line segments constituted a figure and showed virtually no response to stimulus B, the "background" casc. It also responded hardly to stimuli $C$ and $D$, reflecting a strong orientation selectivity in its responses. Cell 125 exhibited a less strong orientation preference and responded to all stimuli. The figure versus back-

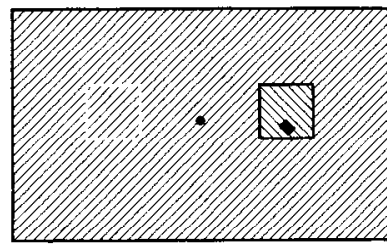

Pos -1.5

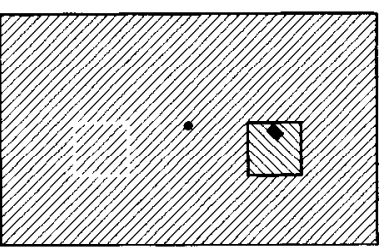

Pos 1.5

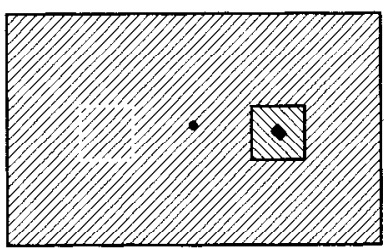

Pos 0.0

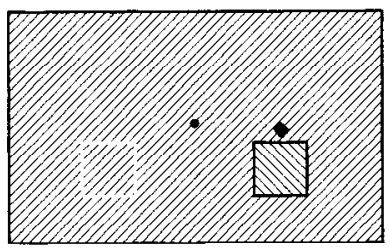

Pos 2.5
Figure 3. Figure windows (black outlined squares) were placed at various positions relative to the receptive field (solid rectangles). The positions of the center of the window are given in degrees of visual angle relative to the receptive field center. 
$\begin{array}{ccc}\text { Cell } 58 & \text { Cell } 87 & \text { Cell } 114 \\ (M l=1.52) & (M \mid=2.45) & (M \mid=2.05)\end{array}$

A
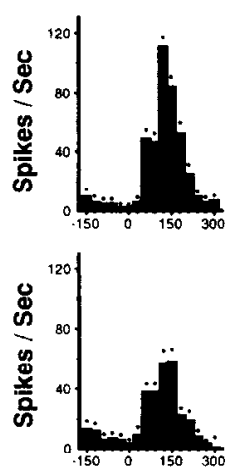

C

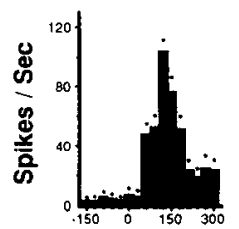

D

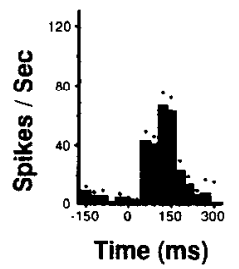

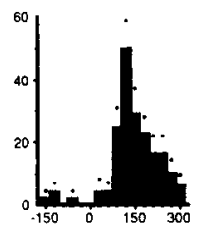
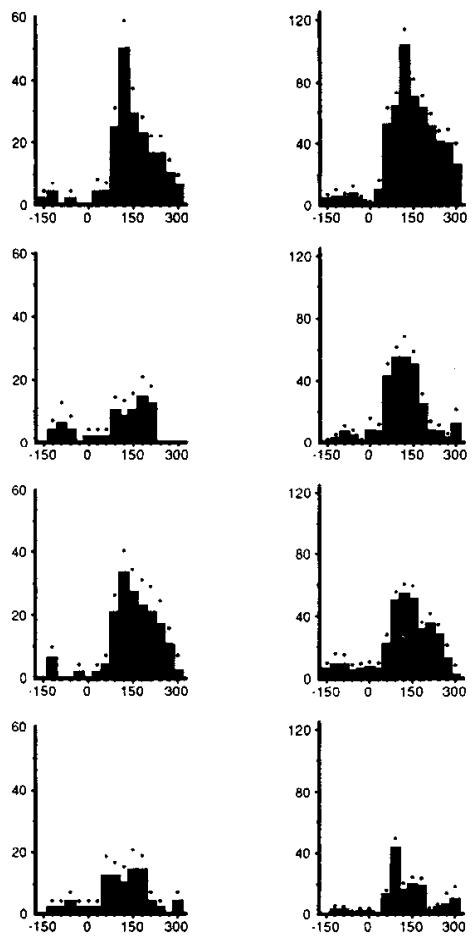

Time (ms)
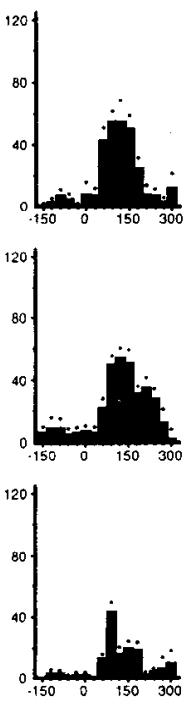

Time (ms)

Figure 4. Three examples of neuronal responses to the random dot motion stimuli of Figure 1. Letters $(4-D)$ correspond to the letters denoting the four stimulus variations in Figure 1 . Bin width of the peristimulus-time histograms (PSTHs) is $30 \mathrm{msec}$. The distance of the dots to the top of each bar gives the SD. $M I$ denotes the modulation index for the given cell.

ground enhancement effect was present for both the preferred ( $C$ and $D)$ and nonpreferred ( $A$ and $B$ ) orientations.

Both monkeys had no difficulties in detecting the position of the motion or orientation defined figures: they made correct saccadic eye movements to these targets in about $95 \%$ of the trials that were completed until "fix-spot off." Performance dropped somewhat when the windows were positioned at larger eccentricities.

When the monkeys were required to make saccades to the red targets instead of to motion or orientation defined figures the results were not different: the responses were equally enhanced for the cases where the figure overlaid the receptive field. This indicates that the response enhancement was not due to selective attention or reward related activity. Experiments with only the background dots or line segments, so leaving the windows blank, elicited no response in all cells where this control was performed. This showed that the background did not encroach on the receptive fields, which is in accordance with the range of receptive field sizes encountered (see Fig. 8C). Experiments with only the window dots or line segments, so leaving the background blank yielded no difference between responses $A$ and $B$ or $C$ and $D$, showing that the effect was not due to data acquisition or analysis crrors or to unrecognized systematic variations in response strength.

Particularly for the moving stimulus, the occurrence of eye movements tracking the background motion might be of some

A
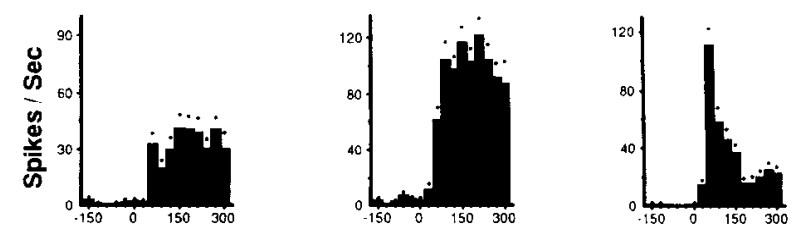

B
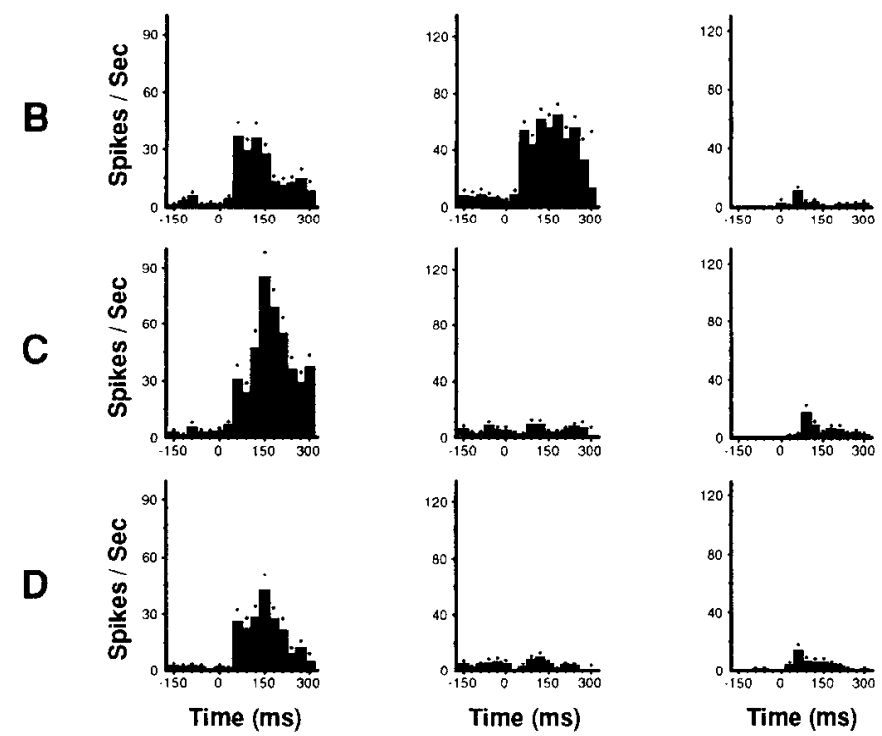

Figure 5. Three examples of neuronal responses to the oriented line segment stimuli of Figure 2. Letters $(A-D)$ correspond to the letters denoting the four stimulus variations in Figure 2 . Bin width of the peristimulus-time histograms (PSTHs) is $30 \mathrm{msec}$. The distance of the dots to the top of each bar gives the SD. MI denotes the modulation index for the given cell.

concern, thereby providing a possible source of artifacts. To exclude this, eye movements during the relevant phase of the trials (from -150 to $300 \mathrm{msec}$ ) were recorded for the four stimulus conditions (A to D) of the motion stimulus. The average eye position and velocity signals were essentially flat for all four conditions (data not shown). However, particularly in the case of tracking or nystagmus like movements that are not tightly locked to stimulus onset, the median of the eye velocity distribution will be a better indication of any deviation from perfect fixation than an averaged signal. Therefore, the distributions of eye velocities along the diagonal of stimulus movement during the interval $0-300 \mathrm{msec}$ were calculated for the four stimulus conditions depicted in Figure 1. Table $1 A$ gives the medians of these distributions. Compared to the stimulus velocity $(1.5 \% \mathrm{sec})$ the eye velocities are very small and close to zero (no strong velocity bias in any direction). Table $1 B$ gives the results of tests for significant differences between the medians. None of the four distributions are significantly different from each other. It can therefore be concluded that eye movements have not contributed in any major way to the results presented here.

\section{Modulation indices}

Above each response set in figures 4 and 5 the modulation index (see Materials and Methods) for the cell is given. A modulation index value higher than 1.0 indicates a larger response for the cases where the window overlying the receptive field was per- 


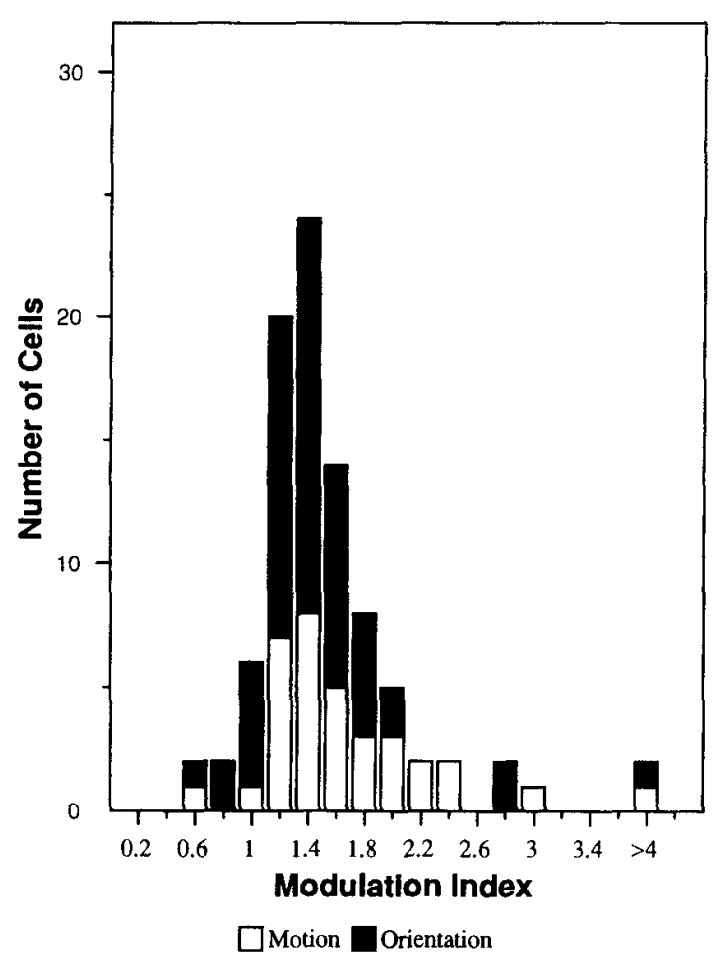

Figure 6. Distribution of figure-ground modulation index values (see Materials and Methods) for all cells recorded from. Bin width is 0.2. The open part of each bar gives the number of cells where the random dot motion stimuli were used, the solid part gives the number of cells for which the oriented line segment stimuli were used. Both stimulus types were used for seven cells.

ceived as the figure. Figure 6 shows the distribution of modulation indices (sce Materials and Methods) for all cells. Thesc modulation indices were obtained with the receptive field in the center of the figure window. Virtually all cells had a modulation index higher than 1.0. The two cells with modulation indices below 0.7 showed an inhibition of their maintained activity after stimulus onset and this inhibition was stronger for the figure case.

Although the figure squares were much larger than the receptive fields studied it could not be ascertained from these results alone whether the observed response enhancements were due to figure-ground segregation. Lateral inhibitions or other effects related to the detection of motion or orientation defined edges might give a similar result. Therefore, the response enhancement was assessed for various (vertical) positions of the windows relative to the receptive fields (see Fig. 3). For each position a modulation index was calculated. Note that all directions of motion or orientations within the windows as well as in the background contribute equally to the sum of responses $A$ and $C$ as to the sum of responses $B$ and $D$. Thercfore, the modulation index could be used as a measure of response enhancement for positions inside as well as outside the square window. Some examples of the resulting graphs, obtained using either the motion or the orientation displays, are shown in Figure 7. Modulation indices were clearly above 1.0 for all positions where the receptive field was within the figure window. However, as soon as the receptive field was outside the window, the index dropped to about 1.0. These data demonstrate that responses to elements forming the figure were uniformly larger along the
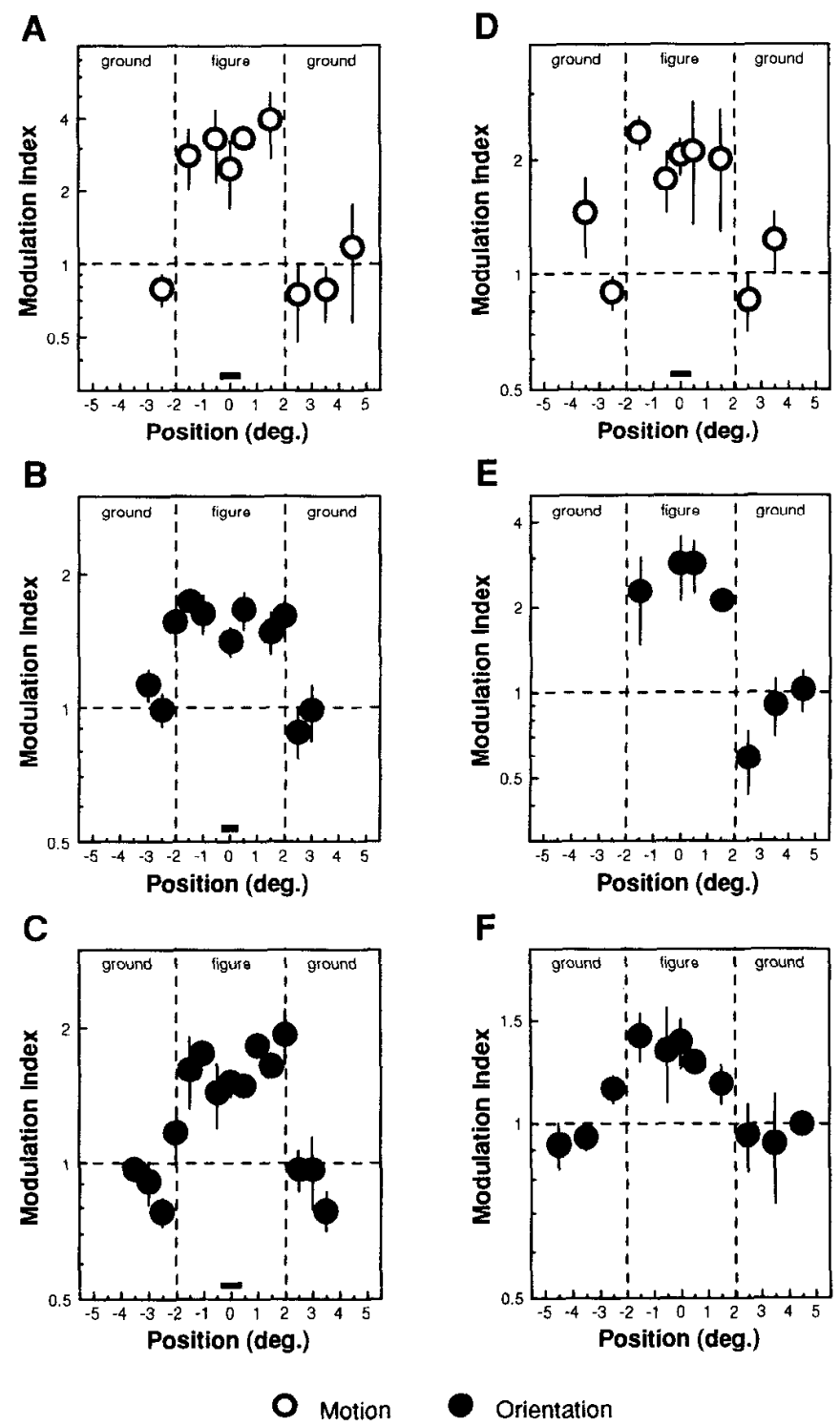

Figure 7. Examples of plots giving figure-ground modulation indices for various positions of the figure window relative to the receptive field (see Fig. 3). Both random dot motion (plots with open circles) and oriented line segment (solid circles) displays were used. Vertical bars give SDs. The two vertical dashed lines in each plot indicate the positions where the border between figure and background is overlying the receptive field. The horizontal dashed line gives the level of modulation index 1.0 , indicating no difference between responses $A+C$ and $B+D$. The single horizontal dash gives the size of the receptive field of the neuron recorded from (when estimated).

entire extent of that figure than the responses elicited by the elements when they formed the background. Moreover, the modulation indices of about 1.0 for positions outside the figure window indicate that responses to background features were identical irrespective of the proximity of an object edge. These results argue strongly against the response enhancement being merely due to lateral inhibitions or other mechanisms responding to the motion or orientation defined edges. In those cases one would have expected an effect which would be equally present at both sides of the boundary between figure and background and which would probably wear off towards the center of the figure. The data, on the contrary, show the response enhance- 
A

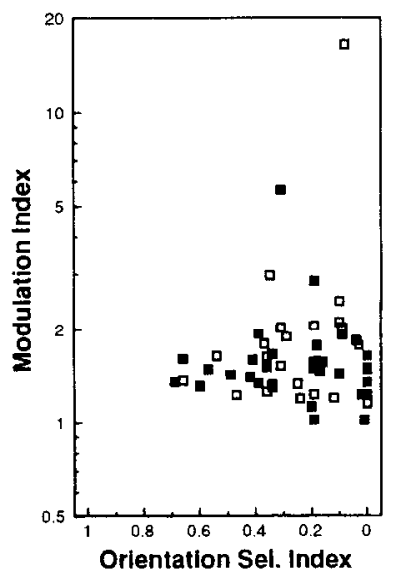

B

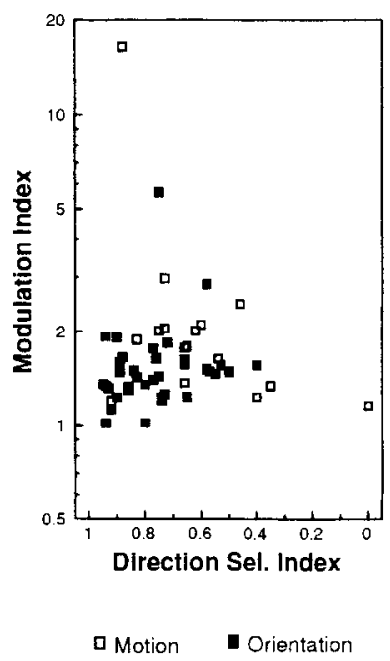

C

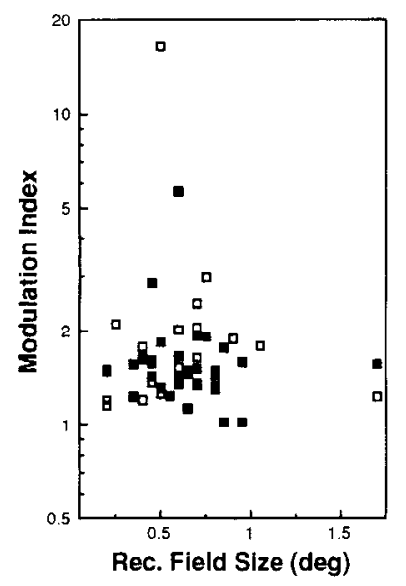

Figure 8. Correlation of figure-ground modulation index with orientation selectivity index $(A)$, direction selectivity index $(B)$, and receptive field size $(C)$. Note the reversed axes of both the orientation and direction selectivity indi$\operatorname{ces}(A$ and $B)$. This is because the lowest value of the index corresponds to the highest degree of selectivity (see Materials and Methods). Open squares give the values for the random dot motion displays; solid squares for the oriented line segment displays.

ment to be highly asymmetrical across the borders of the figure; a strong effect at the "inside edge" and no effect at the "outside edge," in addition to an equally strong effect for the "edge" part of the figure and the center of the figure. In fact, the effect sometimes was even stronger for positions with the receptive field in the center than at the edges (Fig. $7 E, F$ ).

Another peculiarity that was repeatedly observed was that the modulation index was below 1.0 for positions close to the outside edge of the figure and then leveled off to about 1.0 at some larger distance (e.g., Fig. $7 A, D, E$ ). The responses to background elements immediately outside of the figure thus were inhibited relative to other background elements.

\section{Relation of modulation index with receptive field properties}

One might have expected, for example, that the effect for the displays where figure and ground were defined by differences in motion was particularly strong for cells with a high degree of direction selectivity, or that the effect for the oriented line segments displays was strongest for highly orientation selective cells. Therefore, the modulation indices, obtained with the receptive field in the center of the figure window and with both types of displays, were plotted against orientation selectivity (Fig. 8A), direction selectivity (Fig. $8 B$ ), and receptive field size (Fig. $8 C$ ). These plots do not show any correlation between either orientation selectivity, direction selectivity or receptive field size and the modulation indices for either the motion (open squares) or the orientation (solid squares) displays. The receptive field characteristics were admittedly crudely assessed. Nevertheless, a strong correlation, if present, should have left some trace despite this.

\section{Pooled responses and temporal characteristics}

An important question that has not yet been addressed by the results presented thus far pertains to the evolution of the response enhancement over time: what is the latency of the effect with respect to the onset of the neuronal responses itself and is there a difference in latency between responses obtained with the receptive field close to the edge or in the center of the figure? For reasons of signal to noise we analyzed the pooled responses of all cells where data were present for five positions of the receptive field relative to the figure window: $0.5^{\circ}$ outside both edges (pos. $-2.5^{\circ}$ and $\left.+2.5^{\circ}\right), 0.5^{\circ}$ inside both edges (pos. $-1.5^{\circ}$ and $+1.5^{\circ}$ ), and at the center (pos. $0.0^{\circ}$ ) of the figure window.

Figure 9 shows the pooled results for the moving dots display, Figure 10 for the oriented line segments display. The modulation index curves for the pooled responses confirm the results presented earlier: responses were enhanced for elements belonging to the figure.

From the pooled response curves it could be concluded that the figure-background enhancement effect was delayed with respect to the onset of the response of the neurons themselves. This delay was about $40 \mathrm{msec}$ in the motion case and about 30 msec in the orientation case. There was no difference in latency between responses obtained with the receptive field close to the inside edge or in the center of the figure window.

\section{Discussion}

Cells in primary visual cortex of the awake monkey were stimulated with full-screen $\left(32^{\circ} \times 24^{\circ}\right)$ arrays of oriented line segments or moving random dots. A $4^{\circ} \times 4^{\circ}$ square patch of this screen was made to appear as a figure emerging from the background by virtue of differences in the orientations of the line segments or the directions of motion of the dots. The cells almost invariably responded more strongly to elements belonging to the figure than to similar elements belonging to the background. This effect was present throughout the extent of the figure patch and was absent for all positions of the receptive field outside of the figure even when close to the boundary between figure and ground. It appears therefore that the neural image within striate cortex of a stimulus containing a figure within a background consisting of similar elements is a uniform activation of the cells responding to the background elements and a uniform but circa $40 \%$ (mode of distribution of observed effects) higher activation of the cells responding to the figure elements. This enhancement of figure versus background features starts about $30-40 \mathrm{msec}$ after the onset of the neuronal responses itself.

\section{Potential artifacts}

When a moving stimulus is used in an awake monkey recording situation there is always the possibility that tracking eye movements are made in response to the stimulus. In the case of tracking eye movements following the background direction of 

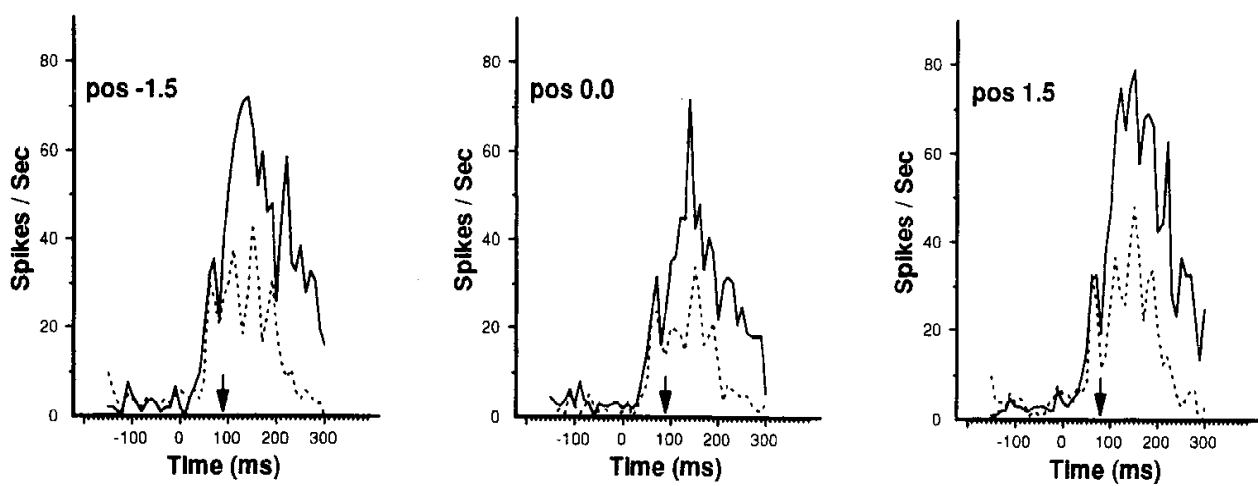

Figure 9. PSTHs of responses pooled from seven neurons for five positions of the random dot motion defined figure window relative to the receptive field center. Solid lines give the PSTH for the case of the figure window within the ipsilateral hemifield, dotted lines for the case of the figure window in the contralateral hemifield. The arrow indicates the first timc instance with a significant difference between the two PSTHs. Bottom row, center, gives a plot of the modulation index versus position for these responses.

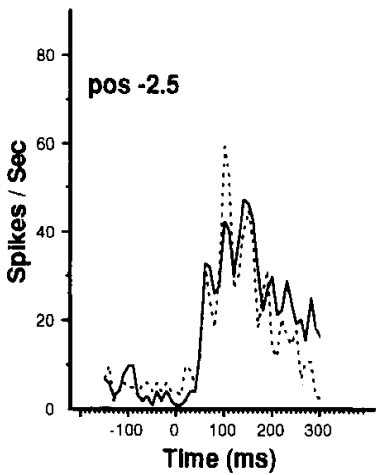

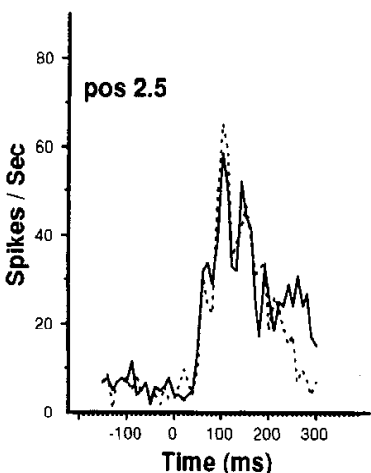

motion a difference between the actual stimulation of the receptive field in cases A and B (or C and D) would have occurred. The results of Table 1 show that there are no significant differences between the eye movements during the four stimulus conditions and that the differences that can be observed are minute compared to the stimulus movement. It seems very unlikely that the figure-ground enhancement effect is caused by eye movements.

When oriented line segments are presented on a video display unit one is faced with the problem that there is a luminance and contrast difference between horizontal and vertical lines. The monitor used in this study was selected for its property to exhibit this effect only in a limited way. After optimal adjustment the luminance difference could be lowered to about $3 \%$. Nevertheless, this means that the "orientation" defined figure-ground displays in the case of horizontal and vertical line segments were also partly "luminance" defined. Of course there was no difference in luminance between lines of both oblique orientations. More than $53 \%$ of our recordings with the oriented line segment stimuli were with oblique line segments and the figure-ground effects were equally present in oblique as well as horizontalvertical displays (Fig. 5, cells 129 and 14, as well as Fig. 7C,E, are examples of results obtained with oblique line segments). The mean modulation index for oblique line segment displays was 1.501 , for horizontal-vertical displays 1.445 . These were not significantly different $[t=0.339, p(\mathrm{H} 0)>>0.20]$. It is

Table 1. A, Median eye velocities during the 0-300 msec interval after stimulus onset for the moving random dot displays of Figure 1. Letters (A-D) correspond to the letters denoting the four stimulus variations in Figure $1(N=4 \times 9600)$. B, Results of median tests, testing for significant differences between the distributions of eye velocities during the four stimulus variations $A-D$. Given are the probabilities of the null hypothesis that the two distributions tested are the same. None of the combinations are significantly different

\begin{tabular}{|l|l|l|l|l|}
\hline Table 1A & A & B & C & D \\
\hline Median & $-0.0058^{\circ} / \mathrm{sec}$. & $0.0103^{\circ} / \mathrm{sec}$. & $0.0015^{\circ} / \mathrm{sec}$. & $-0.0068^{\circ} / \mathrm{sec}$. \\
\hline
\end{tabular}

\begin{tabular}{||l|l|l|l|l|}
\hline Table 1B & A & B & C & D \\
\hline A & $-10.70>p>0.50$ & $0.90>p>0.80$ & $0.98>p>0.95$ \\
\hline B & $0.70>p>0.50$ & - & $0.80>p>0.70$ & $0.80>p>0.70$ \\
\hline C & $0.90>p>0.80$ & $0.80>p>0.70$ & -0.70 & $0.80>p>0.70$ \\
\hline D & $0.98>p>0.95$ & $0.80>p>0.70$ & $0.80>p>0.70$ & $\cdots$ \\
\hline
\end{tabular}



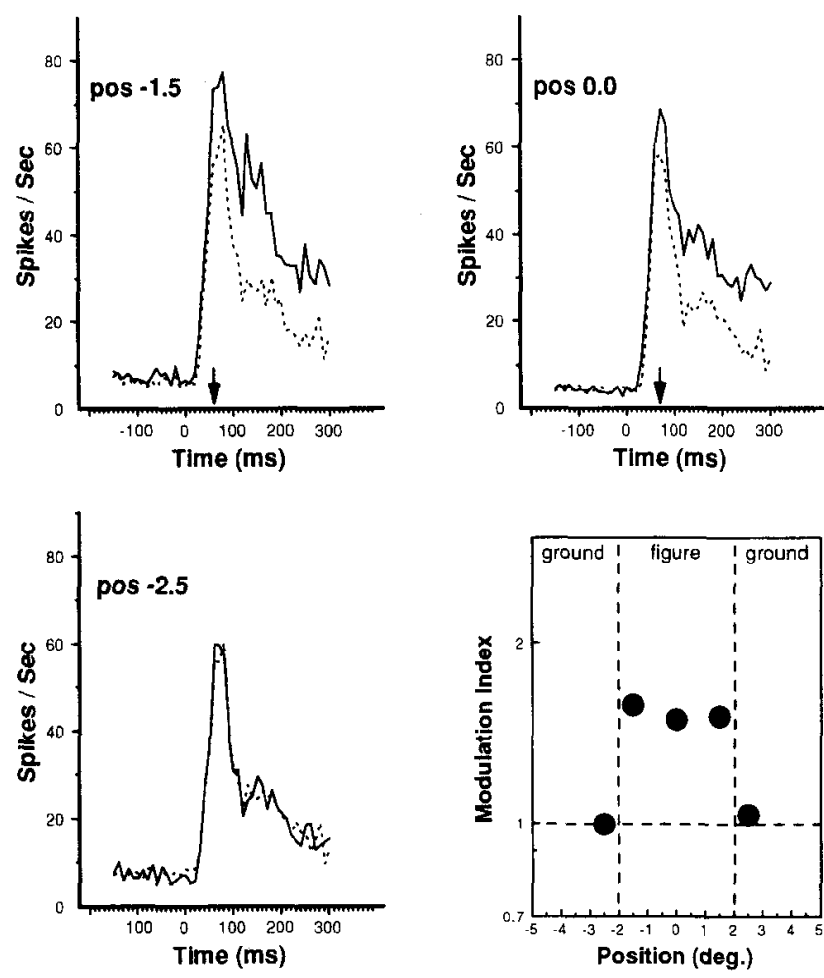

therefore unlikely that the slight luminance difference between horizontal and vertical lines plays any role in the results presented here.

Similar enhancement effects have now also been found for displays where figure and ground are defined by differences in the disparity or the color of textures (Zipser et al,, 1994). Obviously, it would be very difficult to find effects for such a wide range of modalities when they were not truly related to the segregation of figure and ground.

\section{Context modulation}

Primary visual cortical neurons were classically considered to perform a very local analysis of the visual field and therefore would provide only an input to the computational stages involved in the essentially global processes of grouping and segregation. This notion was however based on experiments using simple, localized stimuli. The use of more complex and extensive stimuli indicated that effects on the responses of neurons could be elicited from locations far beyond the receptive field mapped with bars or edges (the "classical" receptive field).

A series of experiments along this line was already performed in the seventies, primarily in cat. Area 17 neurons were stimulated with optimally oriented bars and it was shown that the responses could be either facilitated or inhibited by the presentation of a second bar, sometimes as far away as $24^{\circ}$ but more common within $2^{\circ}$ to $3^{\circ}$. These bars did not produce any response when presented alone (Jones, 1970). Complementary experiments with surrounding bars and gratings showed that inhibition as well as facilitation could be nonspecific or any combination of orientation and direction of motion specific (Maffei and Fiorentini, 1976; Fries et al., 1977; Nelson and Frost, 1978; for a review, see Allman et al., 1985a).

Several experiments specifically analyzed interactions in the motion domain with the use of random textured backgrounds.
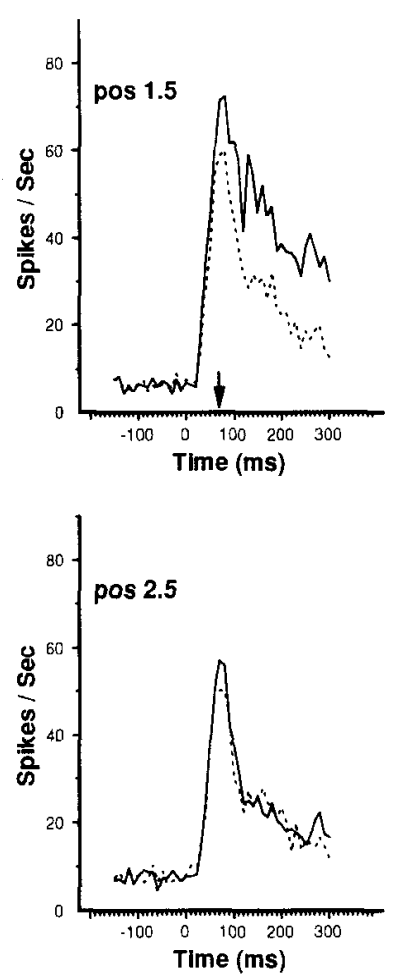

Figure 10. PSTHs of responses pooled from 15 neurons for five positions of the oriented line segment defined window relative to the receptive field center. Bin width is $10 \mathrm{msec}$. Solid lines give the PSTH for the case of the figure window within the ipsilateral hemifield, dotted lines for the case of the figure window in the contralateral hemifield. The arrow indicates the first time instance with a significant difference between the two PSTH's. Bottom row, center, gives a plot of the modulation index versus position for these responses.
Superficial (Hammond and Smith, 1982, 1984) as well as deep layer complex cells (Gulyas et al., 1987; Orban et al., 1987) were reported to change their preferred direction of movement when a background of moving dots was present such that responses were selective for either in-phase or anti-phase motion. Similar observations were reported in the orientation domain. The orientation preference of superficial layer complex cells in the cat was shown to shift away from the orientation of bars presented in the surround (Gilbert and Wiesel, 1990).

Recently, comparable results were obtained in the monkey. Responses to a single bar within the receptive field of striate cortex neurons were shown to be inhibited by the presence of surrounding bars of the same orientation. This inhibition was less strong when the surrounding bars were oriented orthogonally to the center bar (Knierim and Van Essen, 1992). The cells of area V4 of the monkey have been shown to possess very large inhibitory surrounds that even extend into the contralateral hemifield (Desimone et al., 1993). Context modulation was also observed for MT neurons in the motion domain (Allman et al., 1985b) and for V4 neurons in the color domain (Allman et al., 1985a). In conclusion, context modulation appears to be a very general phenomenon throughout the visual brain.

Context modulation is typically discussed in terms like "receptive field surround" or "inhibitory sidebands." In other words, the results are usually interpreted as being caused by some additional receptive field property, albeit one which only reveals itself when the "classical" receptive field is stimulated as well. The results presented here show that this surround has a much more "intelligent" nature than hitherto assumed. The results presented in Figure 7 could never have resulted from some simple inhibitory surround, or even from a receptive field surround that is orientation or direction selective. In those cases the strong asymmetry of the enhancement effect across the border between figure and ground would not have been observed. 
Instead, modulation would have been present on both sides of the border and would probably wear off towards the center of the figure. Even a surround with some non-symmetrical shape cannot explain the findings reported here, where the same asymmetrical figure-ground effect was observed for opposite figureground borders.

In conclusion, the results presented here not only confirm that lateral interactions occur over distances that extend well beyond the classical receptive field, thereby providing contextual information about orientation or direction of motion to the cell. More important, it appears that these interactions create an asymmetry in the processing of figure versus ground which is much like our perception of the visual scene. It is as if the context modulation reflects the (feedback) connections of the cell with the rest of the visual brain, thereby putting the features that cell responds to into their fully evaluated perceptual context, in this case that of a square figure against a background.

\section{Feedback and lateral interactions}

Layers $2 / 3$ and 5 of primary visual cortex are characterized by an abundance of horizontally spreading fibers (Rockland and Lund, 1983) that appear to connect cells with similar orientation tuning over distances of several millimeters (Gilbert and Wiesel, 1989). These connections probably are part of the morphological substrate underlying the context modulation of responses (Lamme et al., 1993a,b), including the effects reported here. However, the figure background enhancement effect appears to operate in a rather "intelligent" way. It would be difficult to envision mechanisms based on simple "local" rules to create the asymmetry across the boundary between figure and background as observed here. Moreover, no correlation was found between orientation or direction selectivity and the strength of the figure-ground enhancement effect up to the point where fully unselective cells showed highly significant effects. This renders it even more unlikely that the process underlying the effect operates on the basis of simple local rules like binding by similarity in feature space.

Another important pathway involved in the processes described here might be the feedback connections from higher visual areas. The delay observed in the figure-ground enhancement effect (see Figs. 9, 10) might allow for feedback from higher visual areas to play a role in the underlying mechanisms. Although feedback connections are just as abundant as feedforward corticocortical connections, little, if anything, is known about their function. Recent theories propose visual processing to be of an iterative nature where feedforward input is transformed through reentrant feedback connections from higher visual areas until some optimum is reached where the most plausible "model" of the outside world is "matched" with the actual input (Mumford, 1992; Tononi et al., 1992; Singer, 1993).

\section{Neuronal synchronization}

The results obtained here neither strongly support nor refute the notion of feature binding by synchronization of neuronal responses (Singer, 1993). They do support the notion, however, that the effects of feature binding are observed in the early stages of processing and that strictly hierarchical models of vision are obsolete. When synchronization is assumed to play an important role in feature binding the results obtained here might be interpreted as an increase in synchronization for figure elements compared to ground elements: increase in synchronization will lead to an increase in response of the involved neurons.
In that case one has to assume that synchronization is a rather "intelligent" process as well and probably involves feedback from higher areas. Thus far, synchronization has mostly been shown to operate along rules of binding by similarity (Gray et al., 1989, 1990; Engel et al., 1990, 1991). The present results suggest that synchronization might work along "higher" principles of perceptual organization as well.

\section{Figure-ground versus attention}

It might be argued that the effects reported here are not due to the neuronal processes underlying the perception of figure and ground but to some modulation caused by selective attention. The figures used here can be considered very strong bottom-up attention grabbers and attention effects have recently been reported for V1 neurons (Motter, 1993). I found similar figureground response enhancements for the two behavioral paradigms used in this study, one of which directed attention away from the motion or orientation defined figures. This indicates that selective attention does not play a major role in the results presented here.

Of course it can not be fully ascertained that the monkeys do not pay any attention to the figure-ground displays when performing the control task. When attention does prove to play a role, it should be concluded from the results discussed above that modulation caused by attention has a very precise spatial localization, effecting only those neurons that are responding to the object attended to. In that case the concepts of focal attention and figure-ground segregation become very confounded.

\section{References}

Allman J, Miezin F, McGuiness E (1985a) Stimulus specific responses from beyond the classical receptive field: neurophysiological mechanisms for local-global comparisons in visual neurons. Annu Rev Neurosci 8:407-430.

Allman J, Miezin F, McGuiness E (1985b) Direction- and velocityspecific responses from beyond the classical receptive field in the middle temporal visual area (MT). Perception 14:105-126.

Barlow HB (1972) Single units and sensation: a neuron doctrine for perceptual psychology? Perception 1:371-394.

Desimone R, Moran J, Schein SJ, Mishkin M (1993) A role for the corpus callosum in visual area V4 of the macaque. Vis Neurosci 10: 159-171.

Eckhorn R, Bauer R, Jordan W, Brosch M, Kruse W, Munk M, Reitboeck HJ (1988) Coherent oscillations: a mechanism of feature linking in the visual cortex? Biol Cybern 60:121-130.

Engel AK, König P, Gray CM, Singer W (1990) Stimulus dependent neuronal oscillations in cat visual cortex: inter-columnar interaction as determined by cross-correlation analysis. Eur J Neurosci 2:588606.

Engel AK, König P, Singer W (1991) Direct physiological evidence for scene segmentation by temporal coding. Proc Natl Acad Sci USA 88:9136-9140.

Fries W, Albus K, Creutzfeldt OD (1977) Effects of interacting visual patterns on single cell responses in cat's striate cortex. Vision Res 17: 1001-1008.

Gilbert CD, Wiesel TN (1989) Columnar specificity of intrinsic horizontal and corticocortical connections in cat visual cortex. J Neurosci 9:2432-2442.

Gilbert CD, Wiesel TN (1990) The influence of contextual stimuli on the orientation selectivity of cells in the primary visual cortex of the cat. Vision Res 30:1689-1701.

Gray CM, König P, Engel AK, Singer W (1989) Oscillatory responses in cat visual cortex exhibit inter-columnar synchronization which reflects global stimulus properties. Nature 338:334-337.

Gray CM, Engel AK, König P, Singer W (1990) Stimulus-dependent neuronal oscillations in cat visual cortex: receptive field properties and feature dependence. Eur J Neurosci 2:607-619. 
Gross CG, Roche-Miranda EC, Bender DB (1972) Visual properties of neurons in inferotemporal cortex of the macaque. J Neurophysiol 35:96-111.

Gulyas B, Orban GA, Duysens J, Maes H (1987) The suppressive influence of moving textured backgrounds on responses of cat striate neurons to moving bars. J Neurophysiol 57:1767-1791.

Ilaenny PR, Schiller PH (1988) State dependent activity in monkey visual cortex. I. Single cell activity in V1 and V4 on visual tasks. Exp Brain Res 69:225-244.

Hammond P, Smith AT (1982) On the sensitivity of complex cells in feline striate cortex to relative motion. Exp Brain Res 47:457-460.

Hammond P, Smith AT (1984) Sensitivity of complex cells in cat striate cortex to relative motion. Brain Res 310:287-298.

Hubel DH, Wiesel TN (1959) Receptive fields of single neurones in the cat's striate cortex. J Physiol (Lond) 148:574-591.

Hubel DH, Wiesel TN (1977) The Ferrier Lecture. Functional architecture of macaque monkey visual cortex. Proc R Soc Lond [Biol] 198:1-59.

Jones BH (1970) Responses of single neurons in cat visual cortex to a simple and a more complex stimulus. Am J Physiol 218:1 102-1 107.

Knicrim JJ, Van Essen DC (1992) Neuronal responses to static texture patterns in area V1 of the alert macaque monkey. J Neurophysiol 67 961-980.

Lamme VAF, Van Dijk BW, Spekreijse H (1993a) Contour from motion processing occurs in primary visual cortex. Nature 363:541543.

Lamme VAF, Van Dijk BW, Spekreijse H (1993b) Organization of texture segregation processing in primate visual cortex. Vis Neurosci $10: 781-790$

Lennie P (1980) Parallel visual pathways: a review. Vision Res 20: 561-594.

Livingstone M, Hubel D (1988) Segregation of form, color, movement, and depth: anatomy, physiology, and perception. Science $240: 740$ 749.

Maffci L, Fiorentini A (1976) The unresponsive regions of visual cortical receptive fields. Vision Res 16:1131-1139.

Maunsell JHR, Newsome WT (1987) Visual processing in monkey extrastriate cortex. Annu Rev Neurosci 10:363-401.

Milner PM (1974) A model for visual shape recognition. Psychol Rev $81: 521-535$
Motter BC (1993) Focal attention produces spatially selective processing in visual cortical areas $\mathrm{V} 1, \mathrm{~V} 2$ and $\mathrm{V} 4$ in the presence of competing stimuli. J Neurophysiol 70:909-919.

Mumford D (1992) On the computational architecture of the neocortex. II. The role of cortico-cortical loops. Biol Cybernetics 66:241251 .

Nelson JI, Frost J (1978) Orientation selective inhibition from beyond the classical visual receptive field. Brain Res 139:359-365.

Orban GA, Gulyas B, Vogels R (1987) Influence of a moving textured background on direction selectivity of cat striate neurons. $J$ Neurophysiol 57:1792-1812.

Perret DI, Mistlin AJ, Chitty AJ (1987) Visual neurones responsive to faces. Trends Neurosci 10:359-364.

Poggio GF, Fischer B (1977) Binocular interaction and depth sensitivity in striate and prestriate cortex of behaving Rhesus monkey. $\mathbf{J}$ Neurophysiol 40:1392-1405.

Robinson DA (1963) A method of measuring eye movement using a scleral search coil in a magnetic field. IEEE Trans Biomed Electron $101: 131$.

Rockland KS, Lund JS (1983) Intrinsic laminar lattice connections in primate visual cortex. J Comp Neurol 216:303-318.

Rolls ET (1991) Neural organization of higher visual functions. Curr Opinion Neurobiol 1:274-278.

Schiller PH, Finlay BL, Volman SF (1976) Quantitative studies of single cell properties in monkey striate cortex. I-V. J Neurophysiol 39:1288-1374.

Schiller PH, Logothetis NK, Charles ER (1990) Role of color- opponent and broad-band channels in vision. Vis Neurosci 5:321-346.

Singer W (1993) Synchronization of cortical activity and its putative role in information processing and learning. Annu Rev Physiol 55: 349-374.

Tononi G, Sporns O, Edelman GM (1992) Reentry and the problem of integrating multiple cortical areas: simulation of dynamic integration in the visual system. Cereb Cortex 2:310-335.

Von der Malsberg C, Schneider W (1986) A neural cocktail-party processor. Biol Cybern 54:29-40.

Zipser K, Lee TS, Lamme VAF, Schiller PH (1994) Invariance of figure-ground mechanisms in Vl for depth, orientation, luminance and chrominance cues. Invest Ophthalmol Vis Sci 35:4(ARVO abstr 3333). 